\title{
Post-operative urothelial recurrence in patients with upper urinary tract urothelial carcinoma managed by radical nephroureterectomy with an ipsilateral bladder cuff: Minimal prognostic impact in comparison with non-urothelial recurrence and other clinical indicators
}

\author{
KIYOSHI TAKAHARA ${ }^{1}$, TERUO INAMOTO ${ }^{1}$, KAZUMASA KOMURA ${ }^{1}$, \\ TOSHIKAZU WATSUII ${ }^{2}$ and HARUHITO AZUMA ${ }^{1}$ \\ ${ }^{1}$ Department of Urology, Osaka Medical College; ${ }^{2}$ Department of Urology, Hirakata City Hospital, Osaka, Japan
}

Received December 4, 2012; Accepted June 20, 2013

DOI: $10.3892 / \mathrm{ol} .2013 .1485$

\begin{abstract}
Upper urinary tract urothelial carcinoma (UTUC) is a rare disease, and novel prognostic factors for patients who have undergone a radical nephroureterectomy (RNU) for UTUC have been studied intensely. To the best of our knowledge, the prognostic value of urothelial recurrence in patients with UTUC has not been previously described in studies. The present study compared the prognostic value of urothelial and non-urothelial recurrence in patients with UTUC of the kidney and ureter managed by surgery. The inclusion criteria consisted of a diagnosis of non-metastatic UTUC (any T stage, N0-1 and M0) and receipt of an RNU with an ipsilateral bladder cuff as the primary treatment. Of the 153 patients that were screened for the study, comprehensive clinical and pathological data was available for 103 patients, who were consequently included in the analysis. Overall survival (OS) and cancer-specific survival (CSS) times were estimated. A multivariate analysis was performed using the Cox regression model. The median follow-up period was 29 months (interquartile range, 14-63 months). The patient population was comprised of 71 males $(68.9 \%)$ and 32 females $(31.1 \%)$. A total of 32 patients (31.1\%) showed non-urothelial recurrence, while 38 patients (36.9\%) exhibited urothelial recurrence and 33 patients $(32.0 \%)$ exhibited no recurrence. When comparing the risk parameters between the non-urothelial recurrence categories, the factors of pathological grade, microvascular invasion, lymphatic invasion and $\mathrm{pT}$ classification showed significant differences. However, there were no significant differences between the urothelial recurrence categories. No significant difference was
\end{abstract}

Correspondence to: Dr Teruo Inamoto, Department of Urology, Osaka Medical College, 2-7 Daigaku-machi, Takatsuki, Osaka 569-8686, Japan

E-mail: tinamoto@poh.osaka-med.ac.jp

Key words: non-urothelial recurrence, upper urinary tract urothelial carcinoma, prognosis observed between the OS and CSS times within the urothelial recurrence categories $(\mathrm{P}=0.3955$ and $\mathrm{P}=0.05891$, respectively), but significant differences were identified in the non-urothelial recurrence categories $(\mathrm{P}<0.0001$ and $\mathrm{P}<0.0001$, respectively). Among the other relevant descriptive pre-operative characteristics in the multivariate analysis, only non-urothelial recurrence remained associated with a worse $\mathrm{CSS}[\mathrm{P}=0.002$; hazard ratio (HR) 9.512]. The results show that urothelial recurrence has a minimal prognostic value in patients with UTUC managed by RNU with an ipsilateral bladder cuff.

\section{Introduction}

Upper urinary tract urothelial carcinoma (UTUC) accounts for $\sim 5 \%$ of all urothelial tumors and $10 \%$ of all renal tumors (1). Since the disease recurrence and progression rates are high in patients with UTUC (2), an improved understanding of the prognostic parameters may lead to the identification of patients who may benefit from intensified therapy and monitoring.

The classical risk factors for the development of UTUC include smoking, abuse of analgesics, chronic urinary tract infection, urolithiasis and oncological agents, such as cyclophosphamide (3). A significant prognostic factor of UTUC is the disease stage. The five-year survival rate for low stage tumors is $\sim 90 \%$, which decreases to $<30 \%$ in cases of regional nodal metastases and to $<10 \%$ in cases of distant metastases (4).

To date, several contemporary, single-center studies of patients who were treated with radical nephroureterectomy (RNU) for UTUC have been published (5-9), and several risk factors for developing UTUC have been reported, including a delay in the RNU (10) and tumor necrosis (11). Although the studies have largely contributed to our understanding of the disease, they were limited by small and heterogeneous populations. To overcome this limitation and to improve our understanding of the natural history of UTUC, a comprehensive database [the Upper Tract Urothelial Carcinoma Collaboration (UTUCC)] incorporating the clinicopathological characteristics and outcomes of $>1,300$ patients treated with RNU for UTUC at 13 academic centers worldwide was created in 
2008 (12). It was concluded that an RNU provided durable local control and cancer-specific survival (CSS) in patients with a localized UTUC, and that the pathological tumor grade, T stage, lymph node status, tumor architecture and lymphovascular invasion (LVI) were significant prognostic variables that were associated with oncological outcomes, which may potentially be used to select patients for adjuvant systemic therapy.

However, there have been no studies that considered the prognostic value of urothelial recurrence in patients with UTUC of the kidney and ureter that is managed by surgery. Consequently, the present study focused on the prognostic impact of urothelial recurrence in comparison with non-urothelial recurrence.

\section{Patients and methods}

Patient selection. The present study was a retrospective analysis of 153 consecutive patients with UTUC, who underwent surgery between 1996 and 2009 at Hirakata City Hospital (Osaka, Japan). Of the 153 patients that were screened for the study, 103 patients were included in the analysis. The inclusion criteria consisted of a diagnosis of non-metastatic UTUC (any T stage, N0-1 and M0) and receipt of an RNU with an ipsilateral bladder cuff as the primary treatment. No patient had an invasive bladder tumor (BT) at the time of the RNU. Written informed consent was obtained from the patient. This study was approved by the ethics committee of Hirakata City Hospital (Osaka, Japan).

In the past, open RNU using an open excision of the distal ureter with a bladder cuff has been performed to dissect the kidney, with the entire length of the ureter and an adjacent segment of the bladder cuff. From June 2003 to date, the approach that has been used is one of conventional four-trocar nephrectomy. Once the nephrectomy is completed, the ureter is dissected and the intact specimen is moved into the pelvis. Next, a semi-Pfannenstiel incision is made in the lower abdomen, which assists in retrieving the specimen, eases the dissection of the lower ureter and facilitates the excision of the bladder cuff. The hilar and regional lymph nodes that are adjacent to the ipsilateral great vessel are then resected, if possible.

Study design. The following clinical and pathological variables were evaluated: Gender, age, tumor side, presence of a BT at diagnosis, serum level of C-reactive protein, hemoglobin, histological type, pathological grade, adjuvant chemotherapy, microvascular invasion, lymphatic invasion, urothelial recurrence, non-urothelial recurrence and pathological stage (2002 TNM system). The tumor grading was assessed according to the 1998 World Health Organization/International Society of Urologic Pathology consensus classification (13). All surgical specimens were processed according to the standard pathological procedures at the Hirakata City Hospital. UTUC was defined as a urothelial carcinoma located in the renal pelvis or calices, as well as tumors located within the ureter. C-reactive protein was pre-operatively obtained from the blood of the UTUC patients and collected in a serum-separating tube. The C-reactive protein level was expressed in units of $\mathrm{mg} / \mathrm{dl}$. Microvascular invasion was defined as tumor cells in an endothelium-lined space observed using routine light microscopy in whole-mounted UTUC specimens. The oncological follow-up schedule included a physical examination, cystoscopy and CT imaging between the chest and pelvis twice per year during the first five years and annually thereafter.

Statistical analysis. The continuous parametric variables were reported as mean $\pm \mathrm{SD}$ and range. The continuous non-parametric variables were presented as median values and interquartile ranges. The $\mathrm{F}$ test was used to assess whether the standard deviations of two populations were equal. $\chi^{2}$ tests were conducted to assess the differences in the covariate distributions between the urothelial and non-urothelial recurrence categories. CSS was defined as the primary endpoint of the study. The survival interval was defined as the time elapsed between the surgery and the last clinical evaluation or cancer-specific mortality. Survival curves were estimated using the Kaplan-Meier method. The patients who remained alive or succumbed to other causes were censored. The log-rank test was used to compare the survival curves. A Cox proportional hazards regression model was used to verify the clinicopathological variables that independently predicted CSS. In all statistical analyses, a two-sided value of $\mathrm{P}<0.05$ was considered to indicate a statistically significant difference. All data were analyzed using the PASW Statistics version 17 statistical program (SPSS Japan Inc., Tokyo, Japan).

\section{Results}

A total of 103 patients with comprehensive clinicopathological data, who fulfilled the inclusion criteria, were included in the analysis (Table I). The mean age was 68.6 years (interquartile range, 62-75 years). During the follow-up period, 45 patients (43.7\%) succumbed to UTUC, 12 (11.7\%) succumbed to other causes and 38 (36.9\%) displayed evidence of disease recurrence. The median follow-up period for the surviving patients was 29 months (interquartile range, 14-63 months). The tumor was located on the right side in 55 patients $(53.4 \%)$ and on the left side in $48(46.6 \%)$. A BT was identified at the time of the UTUC diagnosis in 28 patients $(27.2 \%)$. The histological type was urothelial in 92 patients $(89.3 \%)$ and non-urothelial in $11(10.7 \%)$. The pathological stage was divided into three groups: Superficial (pT0/pTis/pTa/pT1), muscle-invasive (pT2) and non-organ confined (pT3/pT4), which were identified in 43 (41.8\%), 13 (12.6\%) and 47 (45.6\%) patients, respectively.

The median ages of the patients at the time of the surgery in the groups with non-urothelial recurrence $(n=32)$ and without non-urothelial recurrence $(n=71)$ were 70.5 and 69 years, respectively. The median ages in the groups with urothelial recurrence $(n=38)$ and without urothelial recurrence $(n=65)$ were 69 and 71 years, respectively. When comparing the risk parameters between the non-urothelial recurrence categories, the factors of pathological grade, microvascular invasion, lymphatic invasion and $\mathrm{pT}$ classification demonstrated significant differences. However, there were no significant differences observed between the urothelial recurrence categories.

The OS and CSS times between the urothelial recurrence categories showed no significant differences $(\mathrm{P}=0.3955$ and $\mathrm{P}=0.05891$, respectively), while a significant difference was observed within the non-urothelial recurrence categories $(\mathrm{P}<0.0001$ and $\mathrm{P}<0.0001$, respectively; Figs. 1-4).

The univariate analyses using the clinicopathological characteristics, including C-reactive protein, hemoglobin, 
Table I. Clinicopathological characteristics grouped by non-urothelial or urothelial recurrence in 103 patients treated with an RNU and ipsilateral bladder cuff for UTUC.

\begin{tabular}{|c|c|c|c|c|c|c|c|}
\hline \multirow[b]{2}{*}{ Characteristics } & \multirow[b]{2}{*}{ Total } & \multicolumn{3}{|c|}{ Non-urothelial recurrence } & \multicolumn{3}{|c|}{ Urothelial recurrence } \\
\hline & & Yes & No & P-value & Yes & No & P-value \\
\hline Number of patients & 103 & 32 & 71 & & 38 & 65 & \\
\hline Gender, $\mathrm{n}$ & & & & 0.819 & & & 0.511 \\
\hline Male & 71 & 23 & 48 & & 28 & 43 & \\
\hline Female & 32 & 9 & 23 & & 10 & 22 & \\
\hline Age, years & & & & 0.788 & & & 0.138 \\
\hline Mean & 68.6 & 70.3 & 67.8 & & 67.3 & 71.0 & \\
\hline SD & 10.1 & 9.3 & 10.4 & & 10.2 & 9.4 & \\
\hline Median & 69.0 & 70.5 & 69.0 & & 69.0 & 71.0 & \\
\hline Range & $23-91$ & $51-91$ & $23-87$ & & $23-87$ & $54-91$ & \\
\hline Tumor side, $\mathrm{n}$ & & & & 0.400 & & & 0.221 \\
\hline Right & 55 & 15 & 40 & & 17 & 38 & \\
\hline Left & 48 & 17 & 31 & & 21 & 27 & \\
\hline BT at diagnosis, $\mathrm{n}$ & & & & 0.399 & & & 0.255 \\
\hline Yes & 28 & 11 & 17 & & 13 & 15 & \\
\hline No & 75 & 21 & 54 & & 25 & 50 & \\
\hline C-reactive protein $(\mathrm{mg} / \mathrm{ml}), \mathrm{n}$ & & & & 0.282 & & & 0.432 \\
\hline$<0.3$ & 60 & 16 & 44 & & 23 & 37 & \\
\hline$\geq 0.3$ & 43 & 16 & 27 & & 15 & 28 & \\
\hline Hemoglobin $(\mathrm{mg} / \mathrm{ml}), \mathrm{n}$ & & & & 0.599 & & & 0.614 \\
\hline$\leq \mathrm{NR}$ & 75 & 14 & 61 & & 27 & 48 & \\
\hline$>\mathrm{NR}$ & 28 & 18 & 10 & & 11 & 17 & \\
\hline Histological type, $\mathrm{n}$ & & & & 0.166 & & & 0.865 \\
\hline Urothelial & 92 & 24 & 68 & & 32 & 60 & \\
\hline Non-urothelial & 11 & 8 & 3 & & 6 & 5 & \\
\hline Pathological grade, $n$ & & & & 0.008 & & & 0.278 \\
\hline 1 & 20 & 3 & 17 & & 4 & 16 & \\
\hline 2 & 28 & 5 & 23 & & 12 & 16 & \\
\hline 3 & 55 & 24 & 31 & & 22 & 33 & \\
\hline Adjuvant chemotherapy, $\mathrm{n}$ & & & & 0.508 & & & 0.756 \\
\hline Yes & 12 & 5 & 7 & & 5 & 7 & \\
\hline No & 91 & 27 & 64 & & 33 & 55 & \\
\hline Microvascular invasion, $\mathrm{n}$ & & & & 0.001 & & & 0.214 \\
\hline Absent & 69 & 13 & 56 & & 23 & 46 & \\
\hline Present & 34 & 19 & 15 & & 15 & 19 & \\
\hline Lymphatic invasion, $\mathrm{n}$ & & & & 0.005 & & & 0.654 \\
\hline Absent & 71 & 17 & 54 & & 24 & 47 & \\
\hline Present & 32 & 15 & 17 & & 14 & 18 & \\
\hline pT classification, $\mathrm{n}$ & & & & 0.001 & & & 0.209 \\
\hline pT0/pTis/pTa/pT1 & 43 & 6 & 37 & & 15 & 28 & \\
\hline pT2 & 13 & 2 & 11 & & 2 & 11 & \\
\hline pT3/pT4 & 47 & 24 & 23 & & 21 & 26 & \\
\hline
\end{tabular}

RNU, radical nephroureterectomy; UTUC, upper tract urothelial carcinoma; BT, bladder tumor; NR, normal range; pT0/pTis/pTa/pT1, superficial; pT2, muscle invasive; pT3/pT4, non-organ defined.

pathological grade, non-urothelial recurrence, microvascular invasion, lymphatic invasion and $\mathrm{pT}$ classification, were associated with the CSS. In the multivariate analysis for the clinicopathological characteristics, only non-urothelial recurrence was associated with a worse CSS $(\mathrm{P}=0.002$, HR 9.512; Table II). 
Table II. Univariate and multivariate Cox regression models for clinicopathological characteristics predicting CSS in 103 patients treated with RNU and ipsilateral bladder cuff for UTUC.

\begin{tabular}{|c|c|c|c|c|c|c|}
\hline \multirow[b]{2}{*}{ Characteristics } & \multicolumn{3}{|c|}{ Univariate } & \multicolumn{3}{|c|}{ Multivariate } \\
\hline & HR & $95 \% \mathrm{CI}$ & P-value & HR & $95 \% \mathrm{CI}$ & P-value \\
\hline \multicolumn{7}{|l|}{ Gender } \\
\hline Male & Reference & & Reference & & & \\
\hline Female & 0.906 & $0.467-1.758$ & 0.771 & 0.945 & $0.318-2.805$ & 0.919 \\
\hline \multicolumn{7}{|l|}{ Age } \\
\hline Continuous & 1.001 & $0.971-1.033$ & 0.928 & 0.998 & $0.922-1.080$ & 0.954 \\
\hline \multicolumn{7}{|l|}{ Tumor side } \\
\hline Right & Reference & & Reference & & & \\
\hline Left & 0.686 & $0.379-1.241$ & 0.213 & 0.475 & $0.172-1.312$ & 0.151 \\
\hline \multicolumn{7}{|l|}{$\mathrm{BT}$ at diagnosis } \\
\hline Yes & Reference & & Reference & & & \\
\hline No & 1.014 & $0.498-1.952$ & 0.968 & 2.494 & $0.089-1.794$ & 0.232 \\
\hline \multicolumn{7}{|c|}{ C-reactive protein $(\mathrm{mg} / \mathrm{ml})$} \\
\hline Continuous & 1.184 & $1.095-1.281$ & 0.000 & 1.189 & $0.933-1.516$ & 0.161 \\
\hline \multicolumn{7}{|l|}{ Hemoglobin (mg/ml) } \\
\hline Continuous & 1.203 & $0.732-0.944$ & 0.004 & 1.133 & $0.685-1.138$ & 0.337 \\
\hline \multicolumn{7}{|l|}{ Histological type, $\mathrm{n}$} \\
\hline Urothelial & Reference & & Reference & & & \\
\hline Non-urothelial & 2.036 & $0.924-4.486$ & 0.078 & 1.951 & $0.409-9.320$ & 0.402 \\
\hline \multicolumn{7}{|l|}{ Pathological grade, $\mathrm{n}$} \\
\hline $1 / 2$ & Reference & & Reference & & & \\
\hline 3 & 4.918 & $1.984-12.191$ & 0.001 & 2.288 & $0.266-19.705$ & 0.451 \\
\hline \multicolumn{7}{|c|}{ Adjuvant chemotherapy } \\
\hline Yes & Reference & & Reference & & & \\
\hline No & 1.183 & $0.333-2.144$ & 0.723 & 1.499 & $0.149-2.989$ & 0.597 \\
\hline \multicolumn{7}{|l|}{ Urothelial recurrence } \\
\hline No & Reference & & Reference & & & \\
\hline Yes & 0.929 & $0.511-1.690$ & 0.809 & 0.648 & $0.215-1.956$ & 0.442 \\
\hline \multicolumn{7}{|c|}{ Non-urothelial recurrence } \\
\hline No & Reference & & Reference & & & \\
\hline Yes & 5.750 & $3.018-10.954$ & 0.000 & 9.512 & $2.293-39.464$ & 0.002 \\
\hline \multicolumn{7}{|c|}{ Microvascular invasion, $\mathrm{n}$} \\
\hline Absent & Reference & & Reference & & & \\
\hline Present & 8.299 & $3.909-17.618$ & 0.000 & 3.551 & $0.585-21.558$ & 0.168 \\
\hline \multicolumn{7}{|c|}{ Lymphatic invasion, $\mathrm{n}$} \\
\hline Absent & Reference & & Reference & & & \\
\hline Present & 3.953 & $1.966-7.947$ & 0.000 & 1.205 & $0.319-4.553$ & 0.784 \\
\hline \multicolumn{7}{|l|}{ pT classification, $n$} \\
\hline pT0/pTis/pTa/pT1 & Reference & & Reference & & & \\
\hline pT2/pT3/pT4 & 2.619 & $1.676-4.092$ & 0.000 & 0.996 & $0.275-3.611$ & 0.995 \\
\hline
\end{tabular}

CSS, cancer-specific survival; RNU, radical nephroureterectomy; HR, hazard ratio; UTUC, upper tract urothelial carcinoma; pT0/pTis/pTa/pT1, superficial; pT2/pT3/pT4, muscle invasive/non-organ defined.

\section{Discussion}

UTUC is a relatively rare malignancy. Although affected patients may benefit from endoscopic or nephron-sparing approaches, an RNU with an ipsilateral bladder cuff excision remains the standard treatment for patients with large, multifocal or high-grade tumors. However, despite definitive surgery, UTUC remains a malignancy with a high potential for local and distant recurrence, particularly in patients with advanced diseases (14). The outcomes of patients with UTUC following 


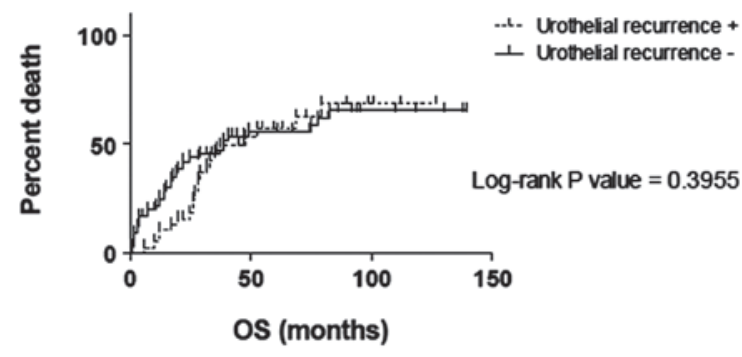

Figure 1. Kaplan-Meier curves of overall survival (OS) time according to the urothelial recurrence categories. Survival curves for the urothelial recurrence-positive (+; broken line) and urothelial recurrence-negative (-; solid line) groups are plotted. The $\mathrm{x}$-axis represents time (months) and the $\mathrm{y}$-axis represents the mortality rate.

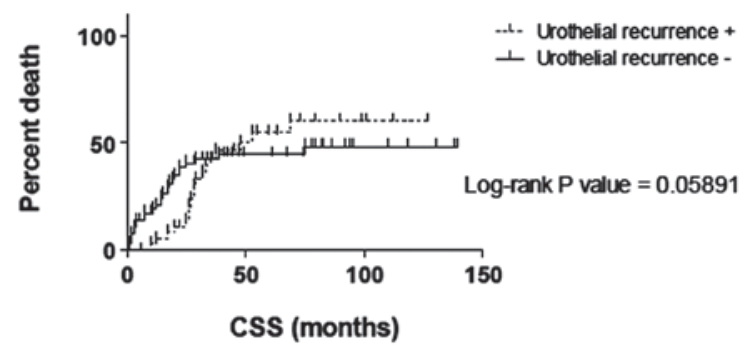

Figure 2. Kaplan-Meier curves of cancer-specific survival (CSS) time according to the urothelial recurrence categories. Survival curves for the urothelial recurrence-positive (+; broken line) and urothelial recurrencenegative (-; solid line) groups are plotted. The x-axis represents time (months) and the y-axis represents the mortality rate. CSS was defined as being alive or succumbing to causes other than cancer. The time period begins at the time of diagnosis and ends at the time of mortality.

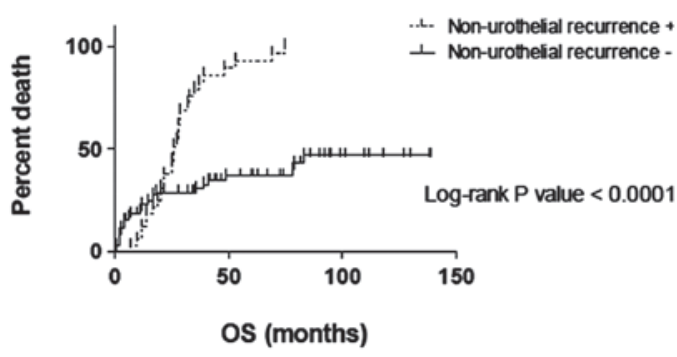

Figure 3. Kaplan-Meier curves of overall survival (OS) time according to the non-urothelial recurrence categories. Survival curves for the non-urothelial recurrence-postive (+; broken line) and non-urothelial recurrence-negative (-; solid line) groups are plotted. The x-axis represents time (months) and the $\mathrm{y}$-axis represents the mortality rate. Results of the log-rank test indicate that OS is significantly higher in the non-urothelial recurrence-negative group $(\mathrm{P}<0.0001)$.

an RNU are heterogeneous and, therefore, difficult to predict. Multi-institutional collaborative studies have identified several potential factors that predict the outcome following an RNU for UTUC, supplementing the traditional pathological staging system (15-17).

Certain papers have examined the prognostic value of urothelial recurrence (particularly intravesical recurrence) following the treatment of an RNU for UTUC. Koda et al reported that intravesical recurrence following surgery for UTUC was not associated with the mode of surgery (i.e. laparoscopy-assisted or open surgery), and that the only risk factor for intravesical recurrence was a history of bladder cancer (18). Several other

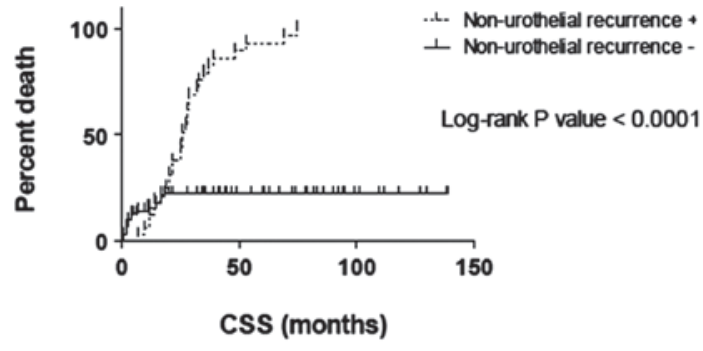

Figure 4. Kaplan-Meier curves of cancer-specific survival (CSS) time according to the non-urothelial recurrence categories. Survival curves for the non-urothelial recurrence-positive (+; broken line) and non-urothelial recurrence-negative (-; solid line) groups are plotted. The $\mathrm{x}$-axis represents time (months) and the $\mathrm{y}$-axis represents the mortality rate. CSS was defined as being alive or succumbing to causes other than cancer. The time period begins at the time of diagnosis and ends at the time of mortality. Results of the log-rank test indicate that CSS is significantly higher in the non-urothelial recurrence-negative group $(\mathrm{P}<0.0001)$

studies reported that it may be important to perform careful follow-up appointments that target intravesical recurrence for patients, particularly males and those with low-stage tumors and/or multifocal tumors, following RNU $(19,20)$. Concomitant carcinoma in situ (CIS) and the tumor size were predictors for bladder cancer recurrence (21). In a series of 196 patients, bladder recurrence was lower in those who received mitomycin $\mathrm{C}$ or epirubicin compared with those who did not received anything (29.0, 25.9 and 41.3\%, respectively) (22). Novara et al observed that only a history of bladder cancer prior to an RNU was an independent risk factor for metachronous recurrence, which was identified in $6 \%$ of patients (23). Youssef et al underlined the prognostic impact of previous bladder cancer (24); patients with a positive bladder cancer (CIS) history had a greater risk of recurrence and mortality from UTUC following RNU (24).

The common locations for the spread of UTUC, depending on the site of the primary tumor, include para-aortic, paracaval, ipsilateral common iliac and pelvic lymph nodes. Hematogenous seeding also occurs in the liver, lungs and bone, which are common sites for metastases. Once a distant metastasis is diagnosed, the prognosis for the patient is extremely poor, in spite of chemotherapy. Certain publications suggest a benefit from the surgical removal of urothelial carcinoma metastases for a subgroup of patients (25). In a large German retrospective study, only 44 patients with distant metastases of the bladder or upper urinary tract underwent a complete resection of all the detectable metastases and were analyzed. The resected metastatic sites included the retroperitoneal lymph nodes (56.8\%), distant lymph nodes $(11.3 \%)$, lung (18.2\%), bone (4.5\%), adrenal gland $(2.3 \%)$, brain $(2.3 \%)$, small intestine $(2.3 \%)$ and skin $(2.3 \%)$. Pre- and/or post-metastasectomy systemic chemotherapy was administered in 35 of 44 patients $(79.5 \%)$. Since no significant prognostic factors were determined due to the limited patient numbers, it was concluded that the metastasectomy in the patients with disseminated urothelial carcinoma metastases remained investigational and there are a limited number of disease types for which a combined-modality approach with systemic chemotherapy would be successful (26).

Lymph node dissection (LND) appears to have an impact on node-positive patients (27). In one previous study, 76 out of 293 patients developed disease relapse. Regional lymph node recurrence was the most common type of relapse (34 patients). 
In the multivariate analyses that adjusted for the effect of tumor stage and grade, $\mathrm{pNx}$ (skipping LND) was an adverse factor for locoregional recurrence and distant relapse (28). However, in the study by Lughezzani et al, which analyzed 2,824 patients from the Surveillance, Epidemiology and End Results (SEER) database, LND showed no benefit in patients with an N0 status compared with those with an Nx status (29). Roscigno et al suggested that LND should be performed in patients with suspected T2-4 stage diseases, to improve the prediction of the natural history of surgically treated UTUC and to use this information for possible adjuvant chemotherapy (30). Thus, the method of defining the right patient, LND template and the extent of the LND remains unclear.

The present study examined the prognostic value of urothelial and non-urothelial recurrence in patients with UTUC of the kidney and ureter that was managed by surgery. The OS and CSS times between the urothelial recurrence categories showed no significant differences, while significant differences were observed in the OS and CSS between the non-urothelial recurrence categories. The factors of pathological grade, microvascular invasion, lymphatic invasion and $\mathrm{pT}$ classification significantly affected the non-urothelial recurrence. However, no factors were observed to significantly affect the urothelial recurrence. In the multivariate analysis for clinicopathological characteristics, only non-urothelial recurrence remained associated with a worse CSS. The present data are limited by the retrospective nature of the study and the relatively small cohort. Prospective studies are required to confirm these findings. However, it may be concluded that non-urothelial recurrence significantly affected the prognosis in patients with UTUC managed by RNU with an ipsilateral bladder cuff compared with those with urothelial recurrence. The findings of the present study underscore the requirement for the careful follow-up and management of urothelial recurrence in patients with UTUC managed by RNU, which aids in lowering the risk of mortality due to cancer.

\section{References}

1. Jemal A, Siegel R, Xu J and Ward E: Cancer statistics, 2010. CA Cancer J Clin 60: 277-300, 2010

2. Margulis V, Youssef RF, Karakiewicz PI, et al; Upper Tract Urothelial Carcinoma Group: Preoperative multivariable prognostic model for prediction of nonorgan confined urothelial carcinoma of the upper urinary tract. J Urol 184: 453-458, 2010.

3. Oosterlinck W, Solsona E, van der Meijden AP, et al; European Association of Urology: EAU guidelines on diagnosis and treatment of upper urinary tract transitional cell carcinoma. Eur Urol 46: 147-154, 2004.

4. Raman JD and Scherr DS: Management of patients with upper urinary tract transitional cell carcinoma. Nat Clin Pract Urol 4: 432-443, 2007.

5. Hall MC, Womack S, Sagalowsky AI, Carmody T, Erickstad MD and Roehrborn CG: Prognostic factors, recurrence, and survival in transitional cell carcinoma of the upper urinary tract: a 30-year experience in 252 patients. Urology 52: 594-601, 1998.

6. Langner C, Hutterer G, Chromecki T, Winkelmayer I, Rehak P and Zigeuner R: pT classification, grade, and vascular invasion as prognostic indicators in urothelial carcinoma of the upper urinary tract. Mod Pathol 19: 272-279, 2006.

7. Novara G, De Marco V, Gottardo F, et al: Independent predictors of cancer-specific survival in transitional cell carcinoma of the upper urinary tract: multi-institutional dataset from 3 European centers. Cancer 110: 1715-1722, 2007.

8. Secin FP, Koppie TM, Salamanca JI, et al: Evaluation of regional lymph node dissection in patients with upper urinary tract urothelial cancer. Int J Urol 14: 26-32, 2007.
9. Brown GA, Busby JE, Wood CG, et al: Nephroureterectomy for treating upper urinary tract transitional cell carcinoma: Time to change the treatment paradigm? BJU Int 98: 1176-1180, 2006.

10. Waldert M, Karakiewicz PI, Raman JD, et al: A delay in radical nephroureterectomy can lead to upstaging. BJU Int 105: 812-817, 2010.

11. Zigeuner R, Shariat SF, Margulis V, et al: Tumour necrosis is an indicator of aggressive biology in patients with urothelial carcinoma of the upper urinary tract. Eur Urol 57: 575-581, 2010.

12. Margulis V, Shariat SF, Matin SF, et al; The Upper Tract Urothelial Carcinoma Collaboration: Outcomes of radical nephroureterectomy: a series from the Upper Tract Urothelial Carcinoma Collaboration. Cancer 115: 1224-1233, 2009.

13. Epstein JI, Amin MB, Reuter VR and Mostofi FK: The World Health Organization/International Society of Urological Pathology consensus classification of urothelial (transitional cell) neoplasms of the urinary bladder. Bladder Consensus Conference Committee. Am J Surg Pathol 22: 1435-1448, 1998.

14. Eng MK and Shalhav AL: Laparoscopic nephroureterectomy: long-term outcomes. Curr Opin Urol 18: 157-162, 2008.

15. Kikuchi E, Margulis V, Karakiewicz PI, et al: Lymphovascular invasion predicts clinical outcomes in patients with node-negative upper tract urothelial carcinoma. J Clin Oncol 27: 612-618, 2009.

16. Remzi M, Haitel A, Margulis V, et al: Tumour architecture is an independent predictor of outcomes after nephroureterectomy: a multi-institutional analysis of 1363 patients. BJU Int 103: 307-311, 2009.

17. Otto W, Shariat SF, Fritsche HM, et al: Concomitant carcinoma in situ as an independent prognostic parameter for recurrence and survival in upper tract urothelial carcinoma: a multicenter analysis of 772 patients. World J Urol 29: 487-494, 2011.

18. Koda S, Mita K, Shigeta M and Usui T: Risk factors for intravesical recurrence following urothelial carcinoma of the upper urinary tract: no relationship to the mode of surgery. Jpn J Clin Oncol 37: 296-301, 2007.

19. Kusuda Y, Miyake H, Terakawa T, Kondo Y, Miura T and Fujisawa M: Gender as a significant predictor of intravesical recurrence in patients with urothelial carcinoma of the upper urinary tract following nephroureterectomy. Urol Oncol: Aug 6, 2011 (Epub ahead of print).

20. Terakawa T, Miyake H, Muramaki M, Takenaka A, Hara I and Fujisawa M: Risk factors for intravesical recurrence after surgical management of transitional cell carcinoma of the upper urinary tract. Urology 71: 123-127, 2008.

21. Pieras E, Frontera G, Ruiz X, Vicens A, Ozonas M and Pizá P: Concomitant carcinoma in situ and tumour size are prognostic factors for bladder recurrence after nephroureterectomy for upper tract transitional cell carcinoma. BJU Int 106: 1319-1323, 2010.

22. Wu WJ, Ke HL, Yang YH, Li CC, Chou YH and Huang CH: Should patients with primary upper urinary tract cancer receive prophylactic intravesical chemotherapy after nephroureterectomy? J Urol 183: 56-61, 2010.

23. Novara G, De Marco V, Dalpiaz O, et al: Independent predictors of contralateral metachronous upper urinary tract transitional cell carcinoma after nephroureterectomy: multi-institutional dataset from three European centers. Int J Urol 16: 187-191, 2009.

24. Youssef RF, Shariat SF, Lotan Y, et al: Prognostic effect of urinary bladder carcinoma in situ on clinical outcome of subsequent upper tract urothelial carcinoma. Urology 77: 861-866, 2011.

25. Remzi M, Shariat S, Huebner W, Fajkovic H and Seitz C: Upper urinary tract urothelial carcinoma: what have we learned in the last 4 years? Ther Adv Urol 3: 69-80, 2011.

26. Lehmann J, Suttmann H, Albers P, et al: Surgery for metastatic urothelial carcinoma with curative intent: the German experience (AUO AB 30/05). Eur Urol 55: 1293-1299, 2009.

27. Bolenz C, Shariat SF, Fernández MI, et al: Risk stratification of patients with nodal involvement in upper tract urothelial carcinoma: value of lymph-node density. BJU Int 103: 302-306, 2009.

28. Abe T, Shinohara N, Muranaka M, et al: Role of lymph node dissection in the treatment of urothelial carcinoma of the upper urinary tract: multi-institutional relapse analysis and immunohistochemical re-evaluation of negative lymph nodes. Eur J Surg Oncol 36: 1085-1091, 2010.

29. Lughezzani G, Jeldres C, Isbarn H, et al: A critical appraisal of the value of lymph node dissection at nephroureterectomy for upper tract urothelial carcinoma. Urology 75: 118-124, 2010.

30. Roscigno M, Shariat SF, Margulis V, et al: Impact of lymph node dissection on cancer specific survival in patients with upper tract urothelial carcinoma treated with radical nephroureterectomy. J Urol 181: 2482-2489, 2009. 